\title{
H. pylori isolates with amino acid sequence polymorphisms as presence of both HtrA- L171 \& CagL-Y58/E59 increase the risk of gastric cancer
}

Yi-Chun Yeh ${ }^{1}$, Hsin-Yu Kuo ${ }^{1,2}$, Wei-Lun Chang ${ }^{1}$, Hsiao-Bai Yang ${ }^{3,4}$, Cheng-Chan Lu ${ }^{3}$, Hsiu-Chi Cheng ${ }^{1,2+}$, Ming-Shiang $\mathrm{Wu}^{6+}$ and Bor-Shyang Sheu ${ }^{1,5^{*+}}$

\begin{abstract}
Background: H. pylori CagL-Y58/E59 increase gastric cancer risk by stronger binding with integrin to faciliate type IV secretory system (T4SS). H. pylori can secrete high temperature requirement A (HtrA) to mediate E-Cadherin cleavage for gastric epithelial junction disruption, so H. pylori CagL can adhere to integrin located on basolateral side of epithelium. The study test whether H. pylori HtrA amino acid polymorphisms can increase gastric cancer risk synergistically with CagL-Y58/E59.

Methods: One-hundred and sixty-four H. pylori-positive patients, including 71 with non-ulcer dyspepsia (NUD), 63 with peptic ulcers (PU), and 30 with gastric cancers (GC), were enrolled to receive upper gastrointestinal endoscopy to obtain gastric biopsies for H. pylori culture and histology by the updated Sydney system. Each isolate was screened for htrA \& cagL genotype by polymerase chain reaction and HtrA \& CagL-Y58/E59 amino acid sequence polymorphisms by sequencing.

Results: The prevalence rates of $h$ trA \& cagL gene were both 100\%. The HtrA amino acid sequence polymorphisms were not different between NUD and PU. The H. pylori isolates of GC had higher rates of HtrA residue 171 as leucine than those of NUD (73.3\% vs. 50.7\%, $P=0.036, \mathrm{OR}[95 \% \mathrm{Cl}]=2.7[1.1-6.8])$. The risk of the $\mathrm{H}$. pylori-infected subjects to get gastric cancer was increased up to 15.4-fold, if the infected isolates had presence of both HtrA-L171 and CagL-Y58/E59 $(P<0.001)$.

Conclusions: The H. pylori isolates of gastric cancer subjects had a higher rate of HtrA-L171. H. pylori isolates with presence of both HtrA-171 \& CagL-Y58/E59 can synergistically increase the risk of gastric cancer.
\end{abstract}

Keywords: Gastric cancer, H. pylori, HtrA, CagL, Type IV secretory system

\section{Introduction}

Helicobacter pylori infection leads to chronic gastritis, peptic ulcers and gastric adenocarcinoma $[1,2]$. H. pylori isolates can display extensive diversities in polymorphisms of virulence factor genes, which may determine an

\footnotetext{
*Correspondence: sheubs@mail.ncku.edu.tw

${ }^{+}$Hsiu-Chi Cheng, Ming-Shiang Wu and Bor-Shyang Sheu contributed equally to this work.

'Department of Internal Medicine, National Cheng Kung University Hospital, College of Medicine, National Cheng Kung University, Tainan, Taiwan

${ }^{5}$ Department of Internal Medicine, Tainan Hospital, Ministry of Health and Welfare, 125 Chuang Shan Road, Tainan, Taiwan

Full list of author information is available at the end of the article
}

increased risk to have gastrointestinal disorders [3-5]. To search the virulence factors of the infected $H$. pylori isolates can thus identify the risky groups for earlier treatment to control the adverse outcome, particularly gastric cancer.

Triple positive cagA-vacA-babA2 H. pylori infection increases the risks of peptic ulcer and gastric cancer in the Western countries [6]. However, in Taiwan, the incidence of triple positive infection is near $100 \%$, so such genomic polymorphisms are not related to the different clinical outcomes [7-10]. CagL, as a component of type VI secretion system (T4SS), binds to integrins $\alpha 5 \beta 1$ at

(c) The Author(s). 2019 Open Access This article is distributed under the terms of the Creative Commons Attribution 4.0 International License (http://creativecommons.org/licenses/by/4.0/), which permits unrestricted use, distribution, and 
the basolateral side of host gastric epitheliums to facilitate CagA translocation for carcinogenesis [11]. The $H$. pylori isolates with CagL amino acid polymorphisms as Y58/E59 exploit higher integrin $\alpha 5 \beta 1$ to carry 4.6-fold risk increase of gastric cancer [4]. Studies analyzed Asian and non-Asian subject cohorts were consistent with our finding to show that CagL-E59 was associated with gastric cancer [12-14]. Moreover, we previous reported H. pylori CagL-Y58/E59 can prime higher integrin $\alpha 5 \beta 1$ in adverse $\mathrm{pH}$ condition to enhance hypochlorhydria vicious cycle for gastric carcinogenesis [15]. However, some results contrasted with those finding in 26,695 and P12 strains $[16,17]$. In addition, It is still nearly $50 \%$ of gastric cancer H. pylori isolates without CagL-Y58/E59, and additional virulence factors to increase risk of gastric cancers are in need of further validation.

High-temperature requirement A $(\mathrm{HtrA})$ protein is a chaperone and serine protease. $H$. pylori HtrA consists of signal domain, serine protease domain, PDZ-1 and PDZ-2 domain $[18,19]$. The secreted HtrA of $H$. pylori opened tight junctions and adherence junctions via cleaving occludin, claudin-8, and the extracellular domain of E-cadherin, and consequently $H$. pylori can across epithelial monolayer to the basolateral membranes to interact with integrin $\alpha 5 \beta 1$ for T4SS injection of CagA $[18,20]$. The residue S221 has been shown as the active site [18, 21]. Moreover, a second hot-spot site is around Q81 according to iPred interface prediction and is confirmed by catalytic activity of Q81A mutation. In addition, some charged residues at the HtrA surface, such as D165, D168 and D260, also display important roles for HtrA activity [22]. Additionally, S164, S166, N208 and K328 are the presumed binding sites of HtrA inhibitor, and their mutations loss proteolytic activity against E-cadherin [19].

The H. pylori isolates from worldwide are highly variable in nucleotide sequence of $h t r A$ gene, and had strain-specific difference in the HtrA cleavage E-cadherin [23]. This study thus validated whether htrA genopositivity or any specific polymorphisms of HtrA amino acid sequence can determine the risk with clinical outcomes after $H$. pylori infection, especially in non CagL-Y58/E59 status. In addition, we validated whether any specific HtrA amino acid sequences synergistically increase the risk of gastric cancer with CagL-Y58/E59. Our data shall be original to suggest HtrA amino acid polymorphisms of $H$. pylori isolates as virulence factor of gastric cancer. The screening strategy for specific HtrA sequences of $H$. pylori isolates will be promising to select risky group for early $H$. pylori eradication to improve gastric cancer control.

\section{Materials \& methods}

\section{Patients and collection of $\mathrm{H}$. pylori isolates}

H. pylori strains were obtained from the gastric biopsy of $H$. pylori-infected patients who underwent upper gastrointestinal endoscopy at National Cheng Kung University Medical Center, Tainan, Taiwan. We proposed the rate of HtrA amino acid polymorphisms in non-cancer strains was $50 \%$. The number of $H$. pylori strains from patients with gastric cancers, non-ulcer dyspepsia, and peptic ulcers was at a 1:2:2 ratio. The total number of strains required was 123 to detect a $30 \%$ difference in the polymorphism rates between cancer strains and non-cancer strains with a two-sided $\alpha$ value of 0.05 and a power of $80 \%(\beta=0.20)$. Assuming a screening failure rate of $20 \%, 148$ strains at least were needed. A total of 164 isolates were obtained from 71 non-ulcer dysplasia (NUD), 63 peptic ulcer (PU), and 30 gastric adenocarcinoma (GC) patients.

In each patient, the endoscopic diagnosis and topographic gastric biopsy for $H$. pylori related pathology were reviewed. Biopsies were stained with haematoxylin and eosin, as well as with modified Giemsa stains, to evaluate the H. pylori-related histological features and to grade severity according to the updated Sydney system [24]. The acute inflammatory score (AIS, range 0-3), chronic inflammation score (CIS, range $0-3$ ), the $H$. pylori density (HPD) for each specimen was scored as our previous studies: in range of $0-5$ for each biopsy specimen.

None of the cases have used with antibiotics or proton pump inhibitor before endoscopy. Each patient has provided blood sampling before endoscopy to obtain serum for pepsinogen and gastrin assay.

\section{H. pylori isolate DNA extraction, PCR and sequencing for htrA \& cagL gene}

Genomic DNA of $H$. pylori was extracted by using the Genomic DNA Purification Kit (ThermoFisher Scientific). The extracted DNA of each isolate was subjected to PCR to amplify the $h$ trA genes using paired primers: htrA_F (5' - GCA TCG GGA TGA TTT TAA CG-3') and htrA_R (5'-AAA CAA CGC TCG TTT GTT TG-3') (Genomics, Taiwan). The PCR mixtures were made in a volume of $50 \mu \mathrm{l}$ containing $200 \mathrm{ng}$ of DNA, $0.2 \mathrm{mM}$ of primers and $25 \mu \mathrm{l}$ of $\mathrm{GoTaq}^{\circ}{ }^{\circ}$ Green Master Mix (Promega, Madison, WI). The PCR reaction was performed with a thermal cycler (2720 thermal cycler, Applied Biosystems, Foster City, CA) under $94^{\circ} \mathrm{C}$ for $5 \mathrm{~min}, 30$ cycles of $94^{\circ} \mathrm{C}$ for 30 $\mathrm{s}, 60^{\circ} \mathrm{C}$ for $30 \mathrm{~s}$, and $72^{\circ} \mathrm{C}$ for $1 \mathrm{~min}$, and then followed by a final elongation step at $72^{\circ} \mathrm{C}$ for $10 \mathrm{~min}$. The mixture was stored at $4^{\circ} \mathrm{C}$. The PCR products were separated by $2 \%$ agarose gel electrophoresis and examined under UV illumination $[4,25]$. The genotype of cagL was applied as the method as used in our previous literature [4].

\section{htrA \& cagL-gnosequencing for translating into amino acid sequences}

The amplified products of the htrA were then subjected to the direct sequencing (Genomics, Taiwan). The nucleotide sequence identities were compared with sequences 
deposited in the GenBank database by BLAST program at the National Center for Biotechnology Information. Amino acid sequences of HtrA were predicted by the standard code, and aligned using GeneDoc (version 2.7.000). The amplified product to assess the CagL-Y58/ E59 was applied as in our previous publication [4]. The 164 htrA and 97 cagL gene sequences were deposited in the GenBank, and the accession numbers were showed in Additional files 1 and 2, respectively.

\section{Serum pepsinogen and gastrin by enzyme-linked immuno-sorbent assay (ELISA)}

Blood samples were collected and centrifuged at 3000 rpm for $15 \mathrm{~min}$ at $4{ }^{\circ} \mathrm{C}$. Sera were obtained and frozen immediately at $-80{ }^{\circ} \mathrm{C}$ until analysis. These collected sera were then measured for the levels of pepsinogen I and II (Biohit Oyj, Helsinki, Finland), and gastrin using ELISA kit (BlueGene Biotech, Shanghai, China) according to the manufacturer's specifications $[15,26]$.

\section{Statistics}

The statistical analysis was performed with the SPSS software (SPSS 12, Chicago, IL, USA). The $x^{2}$ test was used to validate the correlation among gender, $h t r A$ prevalence rates, and amino acid sequence polymorphisms. The $t$ test was used to validate age and serum levels of pepsinogen I, pepsinogen II and gastrin. The Mann-Whitney $U$ test was applied to analyze the differences in histological severity. $P$ value $<0.05$ was considered significant with two-tailed analysis.

\section{Results}

The association of HtrA amino acid polymorphism with the clinical diagnosis

Of the $164 \mathrm{H}$. pylori-positive dyspeptic patients, endoscopic diagnoses included 71 with NUD, 63 with PU, and
30 with GC. The enrolled subjects with GC had higher mean age than those with NUD or PU (59.2 \pm 13.2 vs. 47 . $3 \pm 12.3$ vs. $49.5 \pm 13.2$, respectively, $P<0.001$ ). Males had higher rates of PU than females as compared to NUD subjects $(61.9 \%$ vs. $31 \%, P<0.001)$.

The prevalence of htrA gene of 164 collected isolates was $100 \%$ (). The HtrA amino acid sequence of our isolates had more than $95 \%$ homology. Based on these predicted amino acid sequences, there were 7 residues of HtrA with more than $10 \%$ variation in these isolates, including residue $6 \mathrm{~F} / \mathrm{L}, 25 \mathrm{~N} / \mathrm{S}, 68 \mathrm{~S} / \mathrm{N}, 171 \mathrm{~L} / \mathrm{S}, 303 \mathrm{~V} / \mathrm{I}$, $312 \mathrm{~A} / \mathrm{V}$, and $382 \mathrm{~T} / \mathrm{A}$ or $\mathrm{V}$ (Table 1 ).

Among these 7 variant residues, $6 \mathrm{~F} / \mathrm{L}$ was located at signal domain, $171 \mathrm{~L} / \mathrm{S}$ was located at protease domain, as well as $303 \mathrm{~V} / \mathrm{I}$ and $312 \mathrm{~A} / \mathrm{V}$ were located at PDZ-1 domain (Fig. 1). We analyzed the correlation between HtrA amino acid polymorphisms and clinical diagnosis showed in Table 1. The active sits S211 and functional regulation residues at Q81, S164, D165, S166, D168, N208, D260 and K328 were all conserved among all isolates (Fig. 1).

There were no difference of HtrA amino acid sequence polymorphisms between NUD and PU subjects $(P>0.05)$. However, isolates from GC had significantly higher rates of amino acid sequence 171 as leucine than the isolates from the NUD subjects $(P=0.036$, OR[95\% $\mathrm{CI}]=2.7[1.1-6.8])($ Table 1). There were no difference in the present rate of F6, N25, S68, V303, A312, and T382 between NUD and GC subjects $(P>0.05)$.

\section{Combined HtrA-L171 and CagL-Y58/E59 between gastric cancer and NUD subjects}

Our previous study showed about $50 \%$ gastric cancer isolates with CagL-Y58/E59 sequence, and subjects infected with $H$. pylori with this sequence had increased risk of gastric cancer [4]. However, there were nearly

Table 1 The polymorphisms of HtrA amino acid sequence among isolate from patients with different clinical diagnosis

\begin{tabular}{|c|c|c|c|c|c|}
\hline Residue n (\%) & $\operatorname{NUD}(n=71)$ & $\mathrm{PU}(n=63)$ & $\mathrm{GC}(n=30)$ & $P$ value ${ }^{a}$ OR $(95 \% \mathrm{Cl})$ & $P$ value ${ }^{b}$ OR $(95 \% \mathrm{Cl})$ \\
\hline $6 \mathrm{~F}$ & $40(56.3)$ & $39(61.9)$ & $19(63.3)$ & $\begin{array}{l}0.513 \\
1.3(0.6-2.5)\end{array}$ & $\begin{array}{l}0.515 \\
1.3(0.6-3.2)\end{array}$ \\
\hline $25 \mathrm{~N}$ & $20(28.2)$ & $16(25.4)$ & $11(36.7)$ & $\begin{array}{l}0.718 \\
0.9(0.4-1.9)\end{array}$ & $\begin{array}{l}0.397 \\
1.5(0.6-3.6)\end{array}$ \\
\hline $68 S$ & $11(15.5)$ & $9(14.3)$ & $8(26.7)$ & $\begin{array}{l}0.845 \\
0.9(0.4-2.4)\end{array}$ & $\begin{array}{l}0.189 \\
2.0(0.7-5.6)\end{array}$ \\
\hline $171 \mathrm{~L}$ & $36(50.7)$ & $38(60.3)$ & $22(73.3)$ & $\begin{array}{l}0.264 \\
1.5(0.7-2.9)\end{array}$ & $\begin{array}{l}0.036 \\
2.7(1.1-6.8)\end{array}$ \\
\hline $303 \mathrm{~V}$ & $52(73.2)$ & $48(76.2)$ & $24(80.0)$ & $\begin{array}{l}0.695 \\
1.2(0.5-2.6)\end{array}$ & $\begin{array}{l}0.472 \\
1.5(0.5-4.1)\end{array}$ \\
\hline $312 \mathrm{~A}$ & $44(62.0)$ & 39 (61.9) & 19 (63.3) & $\begin{array}{l}0.994 \\
1.0(0.5-2.0)\end{array}$ & $\begin{array}{l}0.897 \\
1.1(0.4-2.6)\end{array}$ \\
\hline $382 \mathrm{~T}$ & $16(22.5)$ & $11(17.5)$ & $9(30.0)$ & $\begin{array}{l}0.465 \\
0.7(0.3-1.7)\end{array}$ & $\begin{array}{l}0.427 \\
1.5(0.6-3.8)\end{array}$ \\
\hline
\end{tabular}

Abbreviations: NUD non-ulcer dyspepsia, $P U$ peptic ulcer, GC gastric cancer, $O R$ odds ratio, $95 \% C l$ 95\% confidence interval. The $P$ value was determined by $x^{2}$ test. ${ }^{a}$ indicated significance with $P<0.05$ of such parameter between NUD and PU; ${ }^{b}$ between NUD and GC 


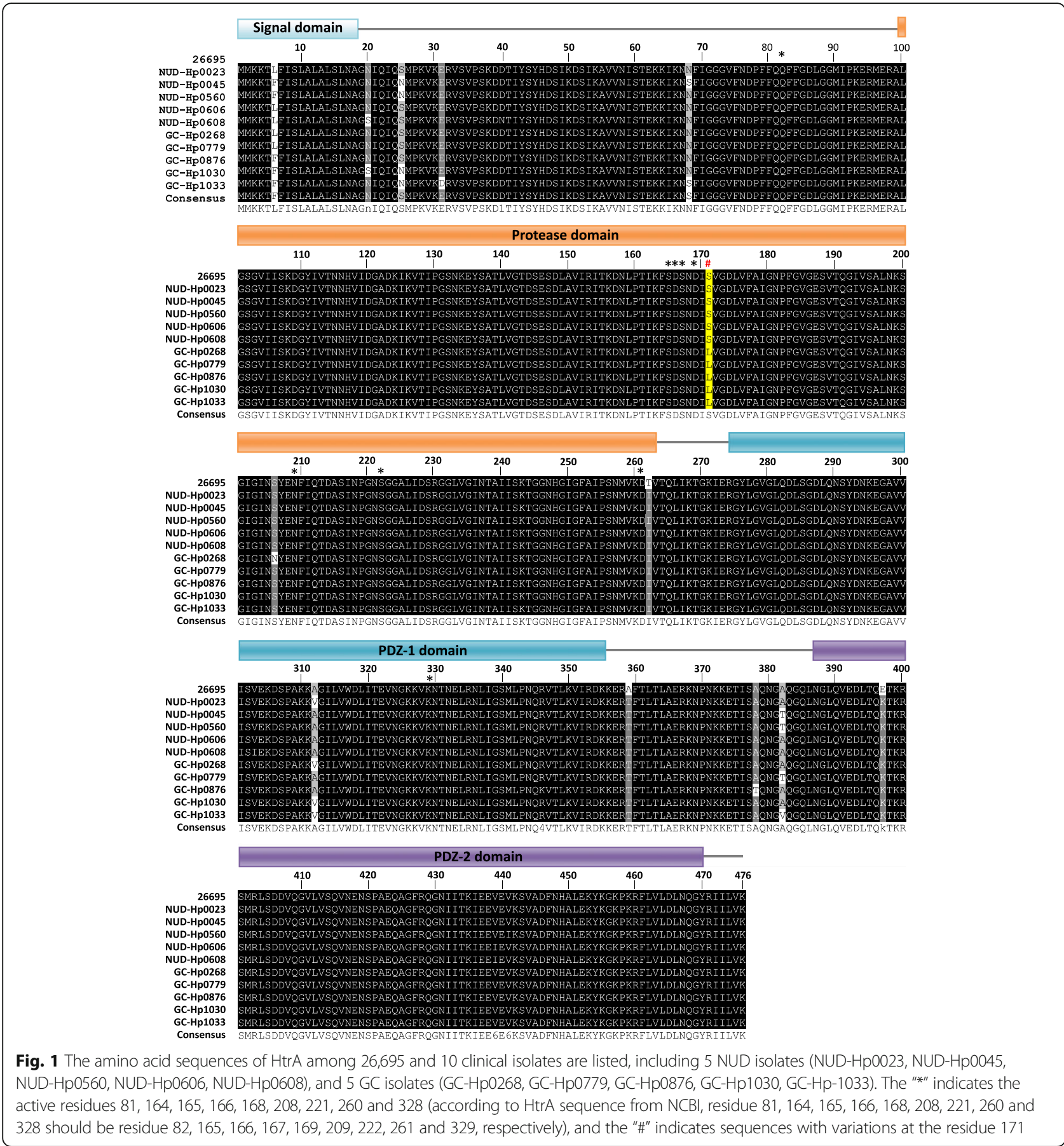

50\% gastric cancer isolates without bearing CagL-Y58/ E59. We thus further examined whether $H$. pylori infection carrying with HtrA-L171 correlates the risk of gastric cancer if subjects infected with a CagL-Y58/E59 absent $H$. pylori.

In Table 2 showed as CagL-Y58/E59 absence, the prevalence of HtrA-L171 was $86.7 \%$ in GC isolates, and significantly higher than in NUD isolates (50.9\%)
$(P=0.013 ;$ OR $[95 \% \mathrm{CI}]=6.3[1.3-30.4])$. We further analyzed whether combined HtrA-L171 and CagL-Y58/ E59 have synergistic effect on the risk of gastric cancer development. In Table 2 , the odds ratio was higher for those infected by $H$. pylori bearing combined HtrA-L171 and CagL-Y58/E59, and the risk could be even higher than as $H$. pylori with HtrA-L171 alone or CagL-Y58/E59 alone. 
Table 2 The comparison of the GC risk as H. pylori bearing with different HtrA-L171 and CagL-Y58/E59 status

\begin{tabular}{llllll}
\hline HtrA-L171 & CagL-Y58/E59 & NUD n (\%) & GC n (\%) & $P$ value & OR (95\% Cl) \\
\hline- & - & $27(49.1)$ & $2(13.3)$ & 0.013 & $6.3(1.3-30.4)$ \\
- & + & $28(50.9)$ & $13(86.7)$ & & \\
- & - & $27(81.8)$ & $2(25.0)$ & 0.002 & $13.5(2.2-84.0)$ \\
+ & - & $6(18.2)$ & $6(75.0)$ & & $15.4(2.7-89.5)$ \\
- & - & $27(79.4)$ & $2(20.0)$ & $<0.001$ & \\
\hline
\end{tabular}

Abbreviations: NUD non-ulcer dyspepsia, GC gastric cancer, OR odds ratio, 95\% Cl 95\% confidence interval. The $P$ value was determined by $x^{2}$ test

Gastric histology in NUD with different H. pylori HtrAL171 \& CagL-Y58/E59 status

Table 3 presented the differences in histological features in the antrum and in the corpus among $H$. pylori infection carrying with different $H$. pylori HtrA-L171 \& CagL-Y58/ E59 status in NUD subjects. Only in the antrum, the CIS was higher in the subjects with $H$. pylori bearing HtrA-L171 alone than in other those infected by $H$. pylori with other HtrA-L171 \& CagL-Y58/E59 status $(P<0.05)$.

\section{Serum gastrin, pepsinogen I \& II of NUD with different HtrA-L171 \& CagL-Y58/E59}

Next we compared the difference of serum markers, including PG I, PG II and gastrin, among NUD subjects with $H$. pylori infection bearing different HtrA-L171 and CagL-Y58/E59 status. In Table 4, the presence of HtrAL171 alone in the $H$. pylori-infected NUD subjects had higher serum gastrin than those infected with double absence of HtrA-L171 \& CagL-Y58/E59 H. pylori isolates $(96.6 \pm 36.8 \mathrm{pg} / \mathrm{ml}$ vs. $34.0 \pm 26.9 \mathrm{pg} / \mathrm{ml}, P=0.003)$.

\section{Discussion}

This study surveyed whether the serine protease HtrA of $H$. pylori correlated with the gastrointestinal disorder, particularly gastric cancers after $H$. pylori infection. The htrA-genopositive prevalence of $H$. pylori isolates were $100 \%$ in Taiwan. Moreover, HtrA active sites were conserved among all $H$. pylori isolates. Our study exhibited that infection of $H$. pylori harboring HtrA-171 as leucine correlated with higher risk of GC. Subjects with such $H$. pylori infection had elevated serum levels of gastrin and more server chronic inflammation in the antrum. Moreover, the risk of the $H$. pylori-infected subjects to get gastric cancer was increased up to 15.4-fold, if the infected isolates had presence of both HtrA-L171 and CagL-Y58/E59.

In the present study, the prevalence of $h t r A$ gene of $H$. pylori isolations from Taiwan was $100 \%$ and consistent with recent report by Tegtmeyer et al. [23]. In addition, our study has applied sequencing to survey the active site 221 and relevant functional regulation sites of HtrA, including residue 81, 164, 165, 166, 168, 208, 260 and 328. Our data showed these residues were conserved in all our isolations and thus confirmed HtrA should be crucial for $H$. pylori survival.

Since the universal presence of HtrA active residues, we further checked whether there was existence of HtrA amino acid sequence polymorphisms to determine the risk of diverse clinical disease outcomes. In Table 1, we demonstrated that isolates bearing HtrA residue L171 had a 2.7-fold risk of gastric cancer development. In our previous report disclosed that $H$. pylori carrying with CagL-Y58/E59 correlated to a 4.6-fold risk of GC. However, still nearly $50 \%$ of GC isolates had absence of

Table 3 The comparison of histopathology among NUD subjects with different status of HtrA-L171 \& CagL-Y58/E59 of H. pylori infection

\begin{tabular}{|c|c|c|c|c|c|}
\hline \multirow{2}{*}{$\begin{array}{l}\text { Histology score, } \\
\text { mean (SD) }\end{array}$} & \multicolumn{4}{|c|}{ HtrA-L171 \& CagL-Y58/E59 status } & \multirow[t]{2}{*}{$P$ value } \\
\hline & Both absence $(n=27)$ & HtrA-171 L alone $(n=28)$ & CagL-Y58/E59 alone $(n=6)$ & Both presence $(n=7)$ & \\
\hline \multicolumn{6}{|l|}{ Antrum } \\
\hline AIS & $1.44(0.97)$ & $1.64(0.78)$ & $1.33(1.03)$ & $1.29(0.95)$ & NS \\
\hline $\mathrm{CIS}$ & $2.74(0.53)$ & $2.96(0.19)$ & $2.67(0.82)$ & $2.71(0.49)$ & $0.039^{\mathrm{a}} ; 0.037^{\mathrm{b}}$ \\
\hline HPD & $3.26(1.26)$ & $3.36(1.19)$ & $3.17(1.72)$ & $3.86(1.07)$ & NS \\
\hline \multicolumn{6}{|l|}{ Corpus } \\
\hline AIS & $1.00(0.96)$ & $0.93(0.98)$ & $1.17(0.98)$ & $0.57(0.98)$ & NS \\
\hline $\mathrm{ClS}$ & $2.37(0.79)$ & $2.32(0.82)$ & $2.83(0.41)$ & $2.14(0.07)$ & NS \\
\hline HPD & $3.41(1.37)$ & $3.46(1.17)$ & $3.67(1.03)$ & $2.43(1.62)$ & NS \\
\hline
\end{tabular}

Abbreviations: AIS acute inflammation score (range 0-3), CIS chronic inflammation score (range 1-3); HPD, H. pylori density (range 0-5). Statistical analysis was performed by Mann-Whitney $\mathrm{U}$ test. ${ }^{\mathrm{a}}$ indicated significance with $\mathrm{P}<0.05$ of such parameter between both absence and HtrA-L171 alone $\mathrm{H}$. pylori infection; ${ }^{\mathrm{b}}$ between HtrA-L171 alone and both presence. NS: no significant difference 
Table 4 The comparison of serum levels of gastrin, PG I and II among NUD subjects with the different status of HtrA-L171 \& CagL-Y58/E59 of H. pylori infection

\begin{tabular}{|c|c|c|c|c|c|}
\hline \multirow{2}{*}{$\begin{array}{l}\text { Parameters, } \\
\text { mean (SD) }\end{array}$} & \multicolumn{4}{|c|}{ HtrA-L171 \& CagL-Y58/E59 status } & \multirow[t]{2}{*}{$P$ value } \\
\hline & Both absence $(n=11)$ & HtrA-171 L alone $(n=7)$ & CagL-Y58/E59 alone $(n=6)$ & Both presence $(n=5)$ & \\
\hline Gastrin (pg/ml) & $34.0(26.9)$ & $96.6(36.8)$ & $60.8(48.0)$ & $54.6(20.7)$ & $0.003^{a}$ \\
\hline PG I (ng/ml) & $105.3(59.2)$ & $112.3(27.7)$ & $116.8(29.0)$ & $99.6(26.1)$ & NS \\
\hline PG II (ng/ml) & $12.2(6.1)$ & $11.5(5.0)$ & $17.4(8.6)$ & $11.7(3.9)$ & NS \\
\hline$P G|/| \mid$ & $9.0(3.2)$ & $10.6(3.2)$ & $7.4(1.8)$ & $9.4(4.2)$ & NS \\
\hline
\end{tabular}

Abbreviations: $P G$ pepsinogen. Statistical analysis was performed by $t$ test. ${ }^{a}$ indicated significance with $P<0.05$ of such parameter between both absence of HtrAL171 \& CagL-Y58/E59 and HtrA-L171 only H. pylori infection. NS: no significant difference

CagL-Y58/E59 [4]. We thus further validate whether HtrA amino acid sequence can be helpful to identify the gastric cancer risk, especially when the $H$. pylori isolates lack CagL-Y58/E59. In this study, we disclosed H. pylori carrying with HtrA-L171 indeed exhibited an increased GC risk as CagL-Y58/E59 absence (Table 2). It reveals that HtrA-L171 has potential as a marker of GC development.

Residue 171 is located at the protease domain of HtrA. Base on the report by Perna and colleagues showing that HtrA mutant S164A, S166A, N208A, and K328A lost their ability to cleave E-cadherin. This observation supports that these residues played relevant roles for the functional regulation of HtrA [19]. L171 is also closed to S164 and S166 (according to NCBI database, S164 and S166 should be S165 and S167 respectively, Fig. 1). Accordingly, it deserves future study to test whether L171 can be a relevant site for the HtrA regulation or whether amino acid change at 171 alters the function of S164 or S166.

Because HtrA mediates disruption of adherence junction via E-cadherin cleavage, $H$. pylori consequently enables to proceed the interaction of $\mathrm{CagL}$ and integrin $\alpha 5 \beta 1$ at basolateral membrane and then to inject effector protein CagA into host epithelium cells [18, 21]. The study thus checked whether combined HtrA-L171 and CagL-Y58/E59 have synergistic risk on GC. In Table 2, the risk of $\mathrm{GC}$ as $H$. pylori carrying with combined HtrA-L171 and CagL-Y58/E59 was higher than as those isolates carrying with HtrA-L171 only or with CagLY58/E59 only. The combined effect from stronger E-cadherin cleavage by HtrA-171 and stronger integrin expression for T4SS by CagL-Y58/E59 may explain the risk increment in part.

We previously elaborated the CagL-Y58/E59 infection with corpus shift of integrin $\alpha 5 \beta 1$ can cause more severe gastric corpus-predominant injury [4]. Corpus-predominant chronic inflammation reduced acid secretion by parietal cell loss, and facilitates carcinogenetic progression [27]. H. pylori with CagL-Y58/E59 can prime more integrin $\alpha 5 \beta 1$ to translocate CagA under hypochlorhydria for gastric carcinogenesis [15]. Unlike
CagL-Y58/E59 H. pylori infection, subjects infected with H. pylori harboring HtrA-L171 displayed more severe gastric antrum-predominant inflammation instead (Table 3). It implies that HtrA-L171 mediating carcinogenesis may differ from the process via the loss of mucosal glands in the corpus.

Subjects with HtrA-L171 H. pylori infection had elevated serum levels of gastrin (Table 4). Gastrin is an important regulator in gastric acid secretion. Moreover, gastrin can regulate the growth and differentiation of gastric epithelial cells, which might be relevant in the pathogenesis of gastric cancer [28]. Increased serum gastrin levels may result from proton pump inhibitors use, atrophic gastritis, and $H$. pylori infection [29, 30]. Wiedemann et al. reported that the interaction of CagL and integrin $\alpha \mathrm{v} \beta 5$ activate gastrin promoter through EGFR/Raf/MAP/Erk signaling cascade [31]. In Table 4, however, there was no significant difference in serum levels of gastrin as H. pylori carrying with CagL-Y58/E59 compared to other groups.

Inge et al., showed that soluble E-cadherin can activate EGFR and downstream ERK1/2 signaling pathway [32]. Because HtrA mediates cleavage of E-cadherin and generates soluble E-cadherin, whether the soluble E-cadherin generated by HtrA may activate EGFR and consequently activate gastrin promoter or mRNA secretion to contribute $\mathrm{GC}$ development deserves further examination.

The limitation to this study is just analysis of isolates from Taiwan. It is in need to further validate in a large number of isolates from Taiwan and other parts, particularly in the western worlds. Furthermore, there is a need to validate whether HtrA-171 polymorphism really exist different ability in protease activity.

\section{Conclusion}

This study showed the Taiwanese H. pylori isolates bearing HtrA-L171 associated with increased risk of GC development, which may work individually or collaborate with CagL-Y58/E59. HtrA-L171 may serve to screen out the risk population for $H$. pylori eradication so as to impede the carcinogenesis process. 


\section{Additional files}

Additional file 1: Accession numbers of $h$ trA gene analyzed in this study. (DOCX $37 \mathrm{~kb}$ )

Additional file 2: Accession numbers of cagL gene analyzed in this study. (DOCX 28 kb)

\section{Abbreviations}

AIS: Acute inflammatory score; CIS: Chronic inflammation score; ELISA: Enzyme-linked immuno-sorbent assay; GC: Gastric cancers; $H$. pylori: Helicobacter pylori; HPD: H. pylori density; HtrA: High temperature requirement A; NUD: Non-ulcer dyspepsia; PCR: Polymerase chain reaction; PU: Peptic ulcers; T4SS: Type IV secretory system

\section{Acknowledgements}

We thanks Ms. Hui-Wen Wu and Ching-Chun Chuang for the clinical assistance to collect samples.

\section{Funding}

The study was supported by the funding from the Ministry of Science and Technology (MOST-104-2314-B-006-025-MY3).

\section{Availability of data and materials}

All data and materials are available.

\section{Authors' contributions}

YCY conducted the HtrA analysis and draft composition. HCC, HYK, and WLC conducted the clinical case collection and endoscopy. HBY analyzed the data and reviewed the histological features. CCL double checked the histology. MSW and BSS coordinated the study design and progress with draft refinement in completion. All authors read and approved the final manuscript.

\section{Ethics approval and consent to participate}

This study was reviewed by Institute Ethnic Review Board (IRB) of National Cheng Kung University Hospital, Tainan, Taiwan.

\section{Consent for publication}

All the individuals had signed consent forms for participating in this research project and use the obtained data in relevant publications.

\section{Competing interests}

The authors declare that they have no competing interests.

\section{Publisher's Note}

Springer Nature remains neutral with regard to jurisdictional claims in published maps and institutional affiliations.

\section{Author details}

${ }^{1}$ Department of Internal Medicine, National Cheng Kung University Hospital, College of Medicine, National Cheng Kung University, Tainan, Taiwan. ${ }^{2}$ Institute of Clinical Medicine, College of Medicine, National Cheng Kung University, Tainan, Taiwan. ${ }^{3}$ Department of Pathology, National Cheng Kung University Hospital, College of Medicine, National Cheng Kung University, Tainan, Taiwan. ${ }^{4}$ Department of Pathology, Ton Yen General Hospital, Hsin-Chu, Taiwan. ${ }^{5}$ Department of Internal Medicine, Tainan Hospital, Ministry of Health and Welfare, 125 Chuang Shan Road, Tainan, Taiwan. ${ }^{6}$ Department of Internal Medicine, National Taiwan University Hospital, College of Medicine, National Taiwan University, Taipei, Taiwan.

Received: 29 May 2018 Accepted: 2 January 2019

Published online: 05 January 2019

\section{References}

1. Labigne A, de Reuse H. Determinants of helicobacter pylori pathogenicity. Infect Agents Dis. 1996;5(4):191-202

2. Makola D, Peura DA, Crowe SE. Helicobacter pylori infection and related gastrointestinal diseases. J Clin Gastroenterol. 2007;41(6):548-58.
3. Sheu BS, Yang HB, Yeh YC, Wu JJ. Helicobacter pylori colonization of the human gastric epithelium: a bug's first step is a novel target for us. J Gastroenterol Hepatol. 2010;25(1):26-32.

4. Yeh YC, Chang WL, Yang HB, Cheng HC, Wu JJ, Sheu BS. H. pylori cagL amino acid sequence polymorphism Y58E59 induces a corpus shift of gastric integrin alpha5beta1 related with gastric carcinogenesis. Mol Carcinog. 2011;50(10):751-9.

5. Cover TL. Helicobacter pylori diversity and gastric cancer risk. MBio. 2016; 7(1):e01869-15.

6. Prinz C, Schoniger M, Rad R, Becker I, Keiditsch E, Wagenpfeil S, Classen M, Rosch T, Schepp W, Gerhard M. Key importance of the helicobacter pylori adherence factor blood group antigen binding adhesin during chronic gastric inflammation. Cancer Res. 2001;61(5):1903-9.

7. Lai CH, Kuo CH, Chen YC, Chao FY, Poon SK, Chang CS, Wang WC. High prevalence of cagA- and babA2-positive helicobacter pylori clinical isolates in Taiwan. J Clin Microbiol. 2002;40(10):3860-2.

8. Chen TS, Chang FY, Lee SD. Smoking and male gender rather than CagA protein are associated with increased risk for duodenal ulcer in helicobacter pylori-infected patients in Taiwan. Dig Dis Sci. 1999;44(10):2076-80.

9. Sheu BS, Sheu SM, Yang HB, Huang AH, Wu JJ. Host gastric Lewis expression determines the bacterial density of helicobacter pylori in babA2 genopositive infection. Gut. 2003;52(7):927-32.

10. Sheu SM, Sheu BS, Yang HB, Li C, Chu TC, Wu JJ. Presence of iceA1 but not cagA, cagC, cagE, cagF, cagN, cagT, or orf13 genes of helicobacter pylori is associated with more severe gastric inflammation in Taiwanese. J Formos Med Assoc. 2002;101(1):18-23.

11. Kwok T, Zabler D, Urman S, Rohde M, Hartig R, Wessler S, Misselwitz R, Berger J, Sewald N, Konig W, et al. Helicobacter exploits integrin for type IV secretion and kinase activation. Nature. 2007;449(7164):862-6.

12. Ogawa H, Iwamoto A, Tanahashi T, Okada R, Yamamoto K, Nishiumi S, Yoshida M, Azuma T. Genetic variants of helicobacter pylori type IV secretion system components CagL and Cagl and their association with clinical outcomes. Gut Pathog. 2017;9:21.

13. Gorrell RJ, Zwickel N, Reynolds J, Bulach D, Kwok T. Helicobacter pylori CagL hypervariable motif: a global analysis of geographical diversity and association with gastric cancer. J Infect Dis. 2016:213(12):1927-31.

14. Cherati MR, Shokri-Shirvani J, Karkhah A, Rajabnia R, Nouri HR. Helicobacter pylori cagL amino acid polymorphism D58E59 pave the way toward peptic ulcer disease while N58E59 is associated with gastric cancer in north of Iran. Microb Pathog. 2017:107:413-8.

15. Yeh YC, Cheng HC, Yang HB, Chang WL, Sheu BS. H. pylori CagL-Y58/E59 prime higher integrin alpha5beta1 in adverse $\mathrm{pH}$ condition to enhance hypochlorhydria vicious cycle for gastric carcinogenesis. PLoS One. 2013; 8(8):e72735

16. Tafreshi M, Zwickel N, Gorrell RJ, Kwok T. Preservation of helicobacter pylori CagA translocation and host cell Proinflammatory responses in the face of CagL Hypervariability at amino acid residues 58/59. PLoS One. 2015;10(7): e0133531.

17. Tegtmeyer N, Lind J, Schmid B, Backert S. Helicobacter pylori CagL Y58/E59 mutation turns-off type IV secretion-dependent delivery of CagA into host cells. PLoS One. 2014;9(6):e97782.

18. Hoy B, Lower M, Weydig C, Carra G, Tegtmeyer N, Geppert T, Schroder P, Sewald N, Backert S, Schneider G, et al. Helicobacter pylori HtrA is a new secreted virulence factor that cleaves E-cadherin to disrupt intercellular adhesion. EMBO Rep. 2010:11(10):798-804.

19. Perna AM, Reisen F, Schmidt TP, Geppert T, Pillong M, Weisel M, Hoy B, Simister PC, Feller SM, Wessler S, et al. Inhibiting helicobacter pylori HtrA protease by addressing a computationally predicted allosteric ligand binding site. Chem Sci. 2014;5:3583-90.

20. Tegtmeyer N, Wessler S, Necchi V, Rohde M, Harrer A, Rau TT, Asche Cl, Boehm M, Loessner $\mathrm{H}$, Figueiredo $\mathrm{C}$, et al. Helicobacter pylori employs a unique basolateral type IV secretion mechanism for CagA delivery. Cell Host Microbe. 2017;22(4):552-60 e555.

21. Lower M, Weydig C, Metzler D, Reuter A, Starzinski-Powitz A, Wessler S, Schneider G. Prediction of extracellular proteases of the human pathogen helicobacter pylori reveals proteolytic activity of the Hp1018/19 protein HtrA. PLoS One. 2008;3(10):e3510

22. Geppert T, Hoy B, Wessler S, Schneider G. Context-based identification of protein-protein interfaces and "hot-spot" residues. Chem Biol. 2011;18(3):344-53.

23. Tegtmeyer N, Moodley $Y$, Yamaoka Y, Pernitzsch SR, Schmidt V, Traverso FR, Schmidt TP, Rad R, Yeoh KG, Bow H, et al. Characterisation of worldwide 
helicobacter pylori strains reveals genetic conservation and essentiality of serine protease HtrA. Mol Microbiol. 2016;99(5):925-44.

24. Dixon MF, Genta RM, Yardley JH, Correa P. Classification and grading of gastritis. The updated Sydney system. International workshop on the histopathology of gastritis, Houston 1994. Am J Surg Pathol. 1996;20(10): 1161-81.

25. Yeh YC, Cheng HC, Chang WL, Yang HB, Sheu BS. Matrix metalloproteinase3 promoter polymorphisms but not dupA-H. pylori correlate to duodenal ulcers in H. pylori-infected females. BMC Microbiol. 2010;10:218.

26. Cheng HC, Tsai YC, Yang HB, Yeh YC, Chang WL, Kuo HY, Lu CC, Sheu BS. The corpus-predominant gastritis index can be an early and reversible marker to identify the gastric cancer risk of helicobacter pylori-infected nonulcer dyspepsia. Helicobacter. 2017;22(4):e12385.

27. Rieder G, Merchant JL, Haas R. Helicobacter pylori cag-type IV secretion system facilitates corpus colonization to induce precancerous conditions in Mongolian gerbils. Gastroenterology. 2005;128(5):1229-42.

28. Smith JP, Nadella S, Osborne N. Gastrin and gastric cancer. Cell Mol Gastroenterol Hepatol. 2017;4(1):75-83.

29. Lamberts R, Creutzfeldt W, Struber HG, Brunner G, Solcia E. Long-term omeprazole therapy in peptic ulcer disease: gastrin, endocrine cell growth, and gastritis. Gastroenterology. 1993;104(5):1356-70.

30. Chuang CH, Sheu BS, Yang HB, Kao AW, Cheng HC, Yao WJ. Hypergastrinemia after helicobacter pylori infection is associated with bacterial load and related inflammation of the oxyntic corpus mucosa. $J$ Gastroenterol Hepatol. 2004;19(9):988-93.

31. Wiedemann T, Hofbaur S, Tegtmeyer N, Huber S, Sewald N, Wessler S, Backert S, Rieder G. Helicobacter pylori CagL dependent induction of gastrin expression via a novel alphavbeta5-integrin-integrin linked kinase signalling complex. Gut. 2012;61 (7):986-96.

32. Inge LJ, Barwe SP, D'Ambrosio J, Gopal J, Lu K, Ryazantsev S, Rajasekaran SA, Rajasekaran AK. Soluble E-cadherin promotes cell survival by activating epidermal growth factor receptor. Exp Cell Res. 2011;317(6):838-48.

Ready to submit your research? Choose BMC and benefit from:

- fast, convenient online submission

- thorough peer review by experienced researchers in your field

- rapid publication on acceptance

- support for research data, including large and complex data types

- gold Open Access which fosters wider collaboration and increased citations

- maximum visibility for your research: over $100 \mathrm{M}$ website views per year

At $\mathrm{BMC}$, research is always in progress.

Learn more biomedcentral.com/submissions 